\title{
Altered expression of E-cadherin and $\beta$-catenin in malignant transformation of sinonasal inverted papillomas*
}

\author{
Bon Seok Koo ${ }^{1}$, Bong Jin Jung ${ }^{1}$, Sun Gui Kim ${ }^{1}$, Zhe Long Liang ${ }^{2}$, \\ Jin-Man Kim², Min-Kyung $\mathrm{Yeo}^{2}$, Ki-Sang Rha ${ }^{1}$ \\ 1 Department of Otolaryngology-Head and Neck Surgery, Cancer Research Institute, Research
Institute for Medical Sciences, Chungnam National University College of Medicine, Daejeon, Korea
2 Department of Pathology, Chungnam National University College of Medicine, Daejeon, Korea
}

SUMMARY

Background: E-cadherin and $\beta$-catenin are molecules that mediate cell-cell adhesion in nor-
mal epithelium. Aberrant expression of these adhesion molecules results in the loss of inter-
cellular adhesion, with possible cell transformation and tumour progression. We determined
the role of E-cadherin and $\beta$-catenin in the pathogenesis of sinonasal inverted papilloma
(IP) and its malignant transformation.

Methods: We determined the expression of E-cadherin and $\beta$-catenin by immunohistochemistry in paraffin-embedded tissue of 21 subjects with nasal polyps, 56 with IPs, 7 IPs with dysplasia and 18 IPs with squamous cell carcinoma (SCC). The clinicopathological variables of the IPs with SCC correlated with the degree of expression of E-cadherin and $\beta$-catenin.

Results: The degree of expression of E-cadherin and $\beta$-catenin in the cell membrane was significantly lower in IPs with SCC than in nasal polyps and IPs. The degree of expression of $\beta$-catenin was significantly lower in IPs with SCC with a malignant proportion $>50 \%$ compared to a malignant proportion $\leq 50 \%$. However, there was no significant association between the degree of expression of E-cadherin and $\beta$-catenin and clinicopathological variables, such as age, gender, T stage, tumour differentiation, or SCC type (metachronous vs. synchronous). In addition, there was no significant relationship between recurrence or survival rate in IPs with SCC and the degree of expression of E-cadherin or $\beta$-catenin in the cell membrane or nuclear $\beta$-catenin.

Conclusion: Decreased expression of E-cadherin and $\beta$-catenin in the cell membrane may be associated with carcinogenesis of IPs and help predict malignant transformation in sinonasal IPs.

Key words: inverted papilloma, squamous cell carcinoma, E-cadherin, $\beta$-catenin, carcinogenesis

\section{INTRODUCTION}

E-cadherin and $\beta$-catenin are cell membrane-associated proteins involved in cell-cell adhesion. The E-cadherin-catenin complex plays a crucial role in epithelial cell-cell adhesion and in the maintenance of tissue architecture ${ }^{(1)}$. Loss of expression or function of the cadherin/catenin complex results in loss of intercellular adhesion, with possible cell transformation and tumour progression ${ }^{(1-3)}$. In recent years, there have been many reports suggesting that modulation of this complex by different mechanisms is an important step in the initiation and progression of human epithelial cancers ${ }^{(4-5)}$. The loss of E-cadherin expression has been shown to promote the transi- tion of well-differentiated adenomas to invasive $\beta$-cell carcinomas in a transgenic mouse model ${ }^{(6)}$. Decreased expression of E-cadherin in lung, prostate, and oesophageal cancers is also associated with poor clinical prognosis ${ }^{(7-10)}$.

Inverted papillomas (IPs) are primarily benign epithelial tumours of the nasal cavities and paranasal sinuses. IPs are characterized by a tendency to recur, a destructive capacity, and the potential for malignant transformation ${ }^{(11)}$. The incidence of malignant changes in individual series of IPs has ranged anywhere from $1 \%-53 \%{ }^{(12,13)}$. Although many studies have been performed on IPs, the mechanisms of malignant 
transformation have not been fully described. The predictive factors for malignant transformation of IPs would benefit clinicians in managing IPs. However, clinical or pathological factors do not appear to be sufficient in predicting malignant associations or transformation of IPs. Moreover, little is known about the relationship between the mechanism of IP transformation and the expression of cell adhesion molecules, such as E-cadherin and $\beta$-catenin in this process, although altered expression of E-cadherin and $\beta$-catenin is associated with carcinogenesis and progression of various human malignancies ${ }^{(14-16)}$. In the current study, we compared the difference in expression of E-cadherin and $\beta$-catenin in nasal polyps (NPs), IPs, IPs with dysplasia, and IPs with squamous cell carcinoma (SCC), and analyzed the correlation between the expression of E-cadherin and $\beta$-catenin and clinicopathological parameters of IPs with SCC.

\section{PATIENTS AND METHODS}

\section{Patients and surgical specimens}

All specimens were harvested from patients treated in the Otorhinolaryngology Department of Chungnam National University Hospital between March 1990 and March 2010. All tissues were fixed in 10\% neutral buffered formalin, embedded in paraffin, and routinely processed for histological examination. One hundred two paraffin-embedded tissue sections, including 21 cases with NPs and 56 with IPs, 7 IPs with dysplasia and 18 IPs with SCC, were obtained from the Department of Pathology. Nasal polyp samples were obtained from patients with chronic rhinosinusitis to serve as controls. Local Institutional Review Board approval was obtained for this study. The average age of patients with NPs (12 males and 9 females) was 41.8 years (range, 10 - 86 years). The average age of patients with IPs (47 males and 9 females) was 51.3 years (range, 16 - 80 years). The average age of patients with IPs with dysplasia (6 males and 1 female) was 58.6 years (range, 32-81 years). The average age of patients with IPs with SCC (15 males and 3 females) was 59.3 years (range, 42 - 86 years). Of the patients with IPs with SCC, 4, 3, 8, and 3 patients were T1, T2, T3, and T4, respectively; 3 and 15 patients had well differentiated and moderately differentiated tumours, while no patients had poorly differentiated tumours. When the percentage of the malignant area within the entire tumour was estimated on representative tissue section slides, 11 patients had tumours with a malignant proportion $>50 \%$ and 7 patients had tumours with a malignant proportion $\leq 50 \%$.

\section{Immunohistochemistry}

All immunohistochemical staining was carried out on formalin-fixed, paraffin-embedded tissue. Sequential tissue sections were cut using a microtome, and set at a thickness of $3 \mu \mathrm{m}$. The tissue sections on the micro-slides were deparaffinized with xylene, hydrated in serial dilutions of alcohol, then retrieval of antigen was conducted using EnVision target retrieval high $\mathrm{pH}$ solution in a Dako PTLink machine (Dako, Glostrup, Denmark) for 30 minutes at $97^{\circ} \mathrm{C}$. Subsequent procedures were conducted at room temperature. Endogenous activities of peroxidase were suppressed by $3 \% \mathrm{H}_{2} \mathrm{O}_{2}$ in methanol for 10 minutes, and 20 minutes of serum-free protein block solution (Dako, Carpenteria, CA, USA) treatment eliminated background staining. The sections were then incubated with primary antibodies for 30 minutes. A mouse monoclonal antibody against human E-cadherin (1:100; Novocastra, Newcastle, UK) and a mouse monoclonal antibody against human $\beta$ - catenin (1:200; BD Biosciences, NJ, USA) were used. After washing slides with TBS, further incubation was performed with EnVision anti-mouse (Dako) polymer for 30 minutes. After rinsing, the chromogen was developed for 5 minutes. The slides were counterstained with Mayer's hematoxylin, dehydrated, and mounted with Canada balsam.

\section{Evaluation of immunohistochemical staining}

The internal positive controls for E-cadherin and $\beta$-catenin consisted of epithelial cells in the NPs. Only membranous staining by E-cadherin and $\beta$-catenin (of any intensity) was evaluated for statistical analysis. The percentage of immunoreactive cells was estimated in 10 random 400(x) microscopic fields. The tissue sections were categorized as $3+, 2+, 1+, 0$, when over $75 \%, 50-75 \%, 10-50 \%$, and $<10 \%$ of the epithelial or tumour cells were positive for immunostaining, respectively. The degree of expression was considered to exhibit 'high expression $(3+\text { and } 2+)^{\prime}$ if greater than $50 \%$ of the epithelial or tumour cells were positive and 'low expression (1+ and 0$)$ ' if less than $50 \%$ of the tumour cells were positive for membranous staining, as described previously ${ }^{(9)}$. For reaching a more precise judgment of positive expression, two pathologists blinded to the stage and patient profiles reviewed the immunohistochemically-stained sections.

\section{Statistical analysis}

SPSS version 14.0 software (SPSS Inc., Chicago, IL, USA) was used for statistical analysis. The significance of E-cadherin and $\beta$-catenin immunoreactivity in the two groups or with different variables was determined using Fisher's exact test and Spearman correlation coefficient. Survival rates were calculated by Kaplan-Meier and log-rank tests. A p-value $<0.05$ was considered statistically significant.

\section{RESULTS}

E-cadherin expression

E-cadherin immunoreactivity in all 21 NPs and in the majority of IPs was shown to have a membranous pattern (Figure 1). All NPs were 3+; low expression was noted in 7 IPs $(1+)$, and high expression in 49 cases $(2+, 7$; and $3+, 42)$. In the 7 IPs with dysplasia, low expression was observed in $3(1+)$ and high expression in $4(2+)$. In the 18 IPs with SCC, low expression was observed in $14(0,7 ; 1+, 7)$, and high expression in $4(2+)$. The percentage of tumours with positive E-cadherin staining was significantly lower in IPs with dysplasia and IPs with SCC than in NPs $(\mathrm{p}<0.05$; Table 1$)$; however, there was no significant difference between IPs and NPs $(p=0.180)$. When compared with IPs, the percentage of tumours with positive E-cadherin staining was significantly lower in IPs with SCC than IPs $(p<$ 
Table 1. E-cadherin immunoreactivity of the cell membrane in nasal polyps, inverted papillomas, inverted papillomas with dysplasia, and inverted papillomas with squamous cell carcinoma.

\begin{tabular}{|c|c|c|c|c|c|c|}
\hline \multirow{3}{*}{ Group } & \multicolumn{4}{|c|}{ E-cadherin expression } & \multirow{3}{*}{ p1-value } & \multirow{3}{*}{ p2-value } \\
\hline & \multicolumn{2}{|c|}{ Low } & \multicolumn{2}{|c|}{ High } & & \\
\hline & 0 & $1+$ & $2+$ & $3+$ & & \\
\hline $\mathrm{NP}(\mathrm{n}=21)$ & & & & 21 & & \\
\hline $\operatorname{IP}(n=56)$ & & 7 & 7 & 42 & 0.180 & \\
\hline $\begin{array}{l}\text { IP with dysplasia } \\
(\mathrm{n}=7)\end{array}$ & & 3 & 4 & & $0.011^{*}$ & 0.073 \\
\hline IP with SCC $(n=18)$ & 7 & 7 & 4 & & $0.000^{*}$ & $0.000^{*}$ \\
\hline
\end{tabular}

NP, nasal polyp; IP, inverted papilloma; SCC, squamous cell carcinoma. p1-value: compared with nasal polyp group, p2-value: compared with inverted papilloma group. ${ }^{*} \mathrm{p}<0.05$ between the two categories for a given variable.

0.001). In IPs with dysplasia, the degree of E-cadherin expression was lower compared with IPs, but there was no statistically significant difference $(\mathrm{p}=0.073$; Table 1$)$.

\section{Beta-catenin expression}

Beta-catenin immunoreactivity in all 21 NPs and the majority of IPs also exhibited a membranous pattern (Figure 2). In a manner similar to E-cadherin staining, the membranous expression of $\beta$-catenin was graded as high or low. All NPs were $3+$. In the 56 patients with IPs, low expression was noted in $1(1+)$, and high expression in $55(2+, 3 ; 3+, 52)$. In the 7 patients with IPs with dysplasia, low expression was observed in $2(1+)$, and high expression in $5(2+)$. In the 18 patients with IPs with SCC, low expression was observed in $10(0,3 ; 1+$, $7)$, and high expression in $8(2+)$. Of the 18 patients with IPs with SCC, 12 had nuclear $\beta$-catenin staining (Figure 3). The percentage of tumours with positive $\beta$-catenin staining was significantly lower in IPs with SCC than in NPs $(\mathrm{p}<0.001$; Table 2); however, there was no significant difference between IPs and NPs $(p=1.000)$. In the IPs with dysplasia, the degree of membranous $\beta$-catenin expression was lower compared to NPs, but there was no statistically significant difference ( $\mathrm{p}=$ 0.056). When compared with IPs, the percentage of tumours with positive $\beta$-catenin staining was significantly lower in IPs with dysplasia and IPs with SCC than IPs ( $<<0.05$; Table 2).

Correlation of E-cadherin and $\beta$-catenin expression with clinicopathologic parameters in inverted papillomas with squamous cell carcinoma

Nine of 10 tumours with low expression of $\beta$-catenin had a malignant proportion $>50 \%$, while only 1 of 8 tumours with high expression of $\beta$-catenin had a malignant proportion $\leq 50 \%$. This showed statistically significant difference $(\mathrm{p}=$ 0.003; Table 3). However, there was no relationship between the degree of expression of $\beta$-catenin and age, gender, tumour stage, tumour differentiation, or SCC type (metachronous vs. synchronous). No significant association existed between the degree of expression of E-cadherin and the clinicopathological variables (Table 3).

Relationship between the expression of E-cadherin and $\beta$-catenin and recurrence or survival in patients with inverted papillomas with squamous cell carcinoma

In patients with IPs with SCC, the follow-up period ranged from 2 - 212 months, with a mean of 74.6 months. During the follow-up, 2 patients $(22.2 \%)$ presented with recurrences and/or metastases. These 2 patients died of disease at 2 and 5 months after primary surgery. The remaining 16 patients were alive without disease. Therefore, the locoregional control rate and disease-specific 5-year survival rate for all 18 patients with IPs with SCC were $88.9 \%$ and $88.5 \%$, respectively. Regarding the relationship between the recurrence or disease-specific survival rates and clinicopathological variables, there were no statistically significant differences in age, gender, tumour stage, tumour differentiation, the degree of malignant proportion, and the degree of expression of E-cadherin or $\beta$-catenin in the cell membrane and nuclear $\beta$-catenin (data not shown).

\section{DISCUSSION}

Inverted papillomas are of epithelial origin and characterized by a hyperplastic epithelium with an endophytic type of growth, while the basement membrane is intact and morphologically normal ${ }^{(17)}$. Inverted papillomas have the potential for malignant transformation. Initially, the nasal columnar (respiratory) epithelium changes into transitional (cuboidal) epithelium, followed by squamous metaplasia. Once epithelial dysplasia is established, carcinoma in situ and invasive SCC may follow ${ }^{(18)}$. Although many studies have been performed on IPs, the factors and mechanisms responsible for malignant transformation are not fully understood.

An intact E-cadherin-catenin complex is required for maintenance of normal intercellular adhesion. E-cadherin, localized to the zonula adherens, complexed with $\beta$ - and $\gamma$-catenin. Beta- 
Table 2. $\beta$-catenin immunoreactivity of the cell membrane in nasal polyps, inverted papillomas, inverted papillomas with dysplasia, and inverted papillomas with squamous cell carcinoma.

\begin{tabular}{|c|c|c|c|c|c|c|}
\hline \multirow{3}{*}{ Group } & \multicolumn{4}{|c|}{$\beta$-catenin expression } & \multirow{3}{*}{ p1-value } & \multirow[b]{3}{*}{ p2-value } \\
\hline & \multicolumn{2}{|c|}{ Low } & \multicolumn{2}{|c|}{ High } & & \\
\hline & 0 & $1+$ & $2+$ & $3+$ & & \\
\hline$N P(n=21)$ & & & & 21 & & \\
\hline $\mathrm{IP}(\mathrm{n}=56)$ & & 1 & 3 & 52 & 1.000 & \\
\hline IP with dysplasia $(n=7)$ & & 2 & 5 & & 0.056 & $0.030^{*}$ \\
\hline IP with SCC $(n=18)$ & 3 & 7 & 8 & & $0.000^{*}$ & $0.000^{*}$ \\
\hline
\end{tabular}

NP, nasal polyp; IP, inverted papilloma; SCC, squamous cell carcinoma. p1-value: compared with nasal polyp group, p2-value: compared with inverted papilloma group. ${ }^{*} \mathrm{p}<0.05$ between the two categories for a given variable.

Table 3. Correlation of E-cadherin and $\beta$-catenin expression with clinicopathological parameters in inverted papillomas with squamous cell carcinoma $(\mathrm{n}=18)$.

\begin{tabular}{|c|c|c|c|c|c|c|}
\hline \multirow[t]{2}{*}{ Variable } & \multicolumn{2}{|c|}{ E- cadherin expression } & \multicolumn{4}{|c|}{$\beta$-catenin expression } \\
\hline & $\begin{array}{c}\text { Low } \\
(\mathrm{n}=14)\end{array}$ & $\begin{array}{l}\text { High } \\
(n=4)\end{array}$ & p-value & $\begin{array}{c}\text { Low } \\
(\mathrm{n}=10)\end{array}$ & $\begin{array}{l}\text { High } \\
(\mathrm{n}=8)\end{array}$ & p-value \\
\hline Age & & & 1.000 & & & 0.321 \\
\hline$\geq 55(\mathrm{n}=12)$ & 9 & 3 & & 8 & 4 & \\
\hline$<55(\mathrm{n}=6)$ & 5 & 1 & & 2 & 4 & \\
\hline Gender & & & 1.000 & & & 0.069 \\
\hline Male $(\mathrm{n}=15)$ & 11 & 4 & & 10 & 5 & \\
\hline Female $(\mathrm{n}=3)$ & 3 & 0 & & 0 & 3 & \\
\hline T stage & & & 1.000 & & & 0.630 \\
\hline $\mathrm{T} 1-2(\mathrm{n}=7)$ & 6 & 1 & & 3 & 4 & \\
\hline T3-4 $(\mathrm{n}=11)$ & 8 & 3 & & 7 & 4 & \\
\hline Histologic grade & & & 1.000 & & & 1.000 \\
\hline Well $(\mathrm{n}=3)$ & 3 & 0 & & 2 & 1 & \\
\hline Moderate $(n=15)$ & 11 & 4 & & 8 & 7 & \\
\hline Malignant proportion & & & 0.588 & & & $0.003^{*}$ \\
\hline$>50 \%(\mathrm{n}=10)$ & 7 & 3 & & 9 & 1 & \\
\hline$\leq 50 \%(\mathrm{n}=8)$ & 7 & 1 & & 1 & 7 & \\
\hline Carcinoma type & & & 0.569 & & & 1.000 \\
\hline Synchronous $(\mathrm{n}=12)$ & 10 & 2 & & 7 & 5 & \\
\hline Metachronous $(n=6)$ & 4 & 2 & & 3 & 3 & \\
\hline
\end{tabular}

${ }^{*} \mathrm{p}<0.05$ between the two categories for a given variable.

and $\gamma$-catenin in turn bind to $\alpha$-catenin, which attaches to actin filaments in the cell cytoskeleton ${ }^{(5)}$. This linkage between transmembranous cadherins and actin filaments of the cytoskeleton is necessary to form strong cell-cell adhesion. E-cadherin is thought to function as a tumour suppressor because it suppresses invasion and metastasis ${ }^{(4)}$. Although, $\beta$-catenin is important in cell adhesion, it is also a molecule that regulates signal transduction. Beta-catenin interacts with the adenomatous polyposis coli (APC) protein in the cytoplasm. The APC protein regulates the level of free $\beta$-catenin in the cell. Betacatenin has the ability to enter the nucleus from the cytoplasm and interact with transcription factors that are involved in inhibiting apoptosis and promoting cellular proliferation and migration, and include the c-myc oncogene and one of the cell cycle regulators (cyclin D1) ${ }^{(5)}$. This protein complex has been implicated in carcinogenesis because its expression is frequently lost in human epithelial cancers ${ }^{(1-5)}$. Recently, immunohistochemical studies in human cancers have frequently shown that a proportion of invasive carcinoma and carcinoma in situ show aberrant levels of E-cadherin and/or catenin expression in comparison to their related normal tissue ${ }^{(14-16)}$. Perl et al. ${ }^{(6)}$ also demonstrated that the loss of E-cadherin mediated cell-cell adhesion is causally involved in the transition from adenoma to invasive carcinoma. Many reports have shown that a change in 


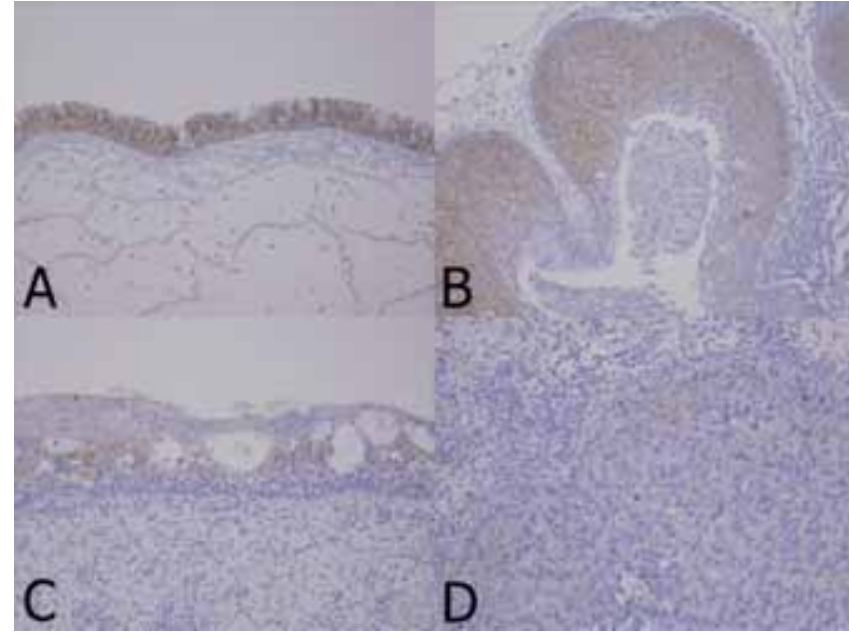

Figure 1. Expression of E-cadherin in nasal polyps (A), inverted papillomas (B), inverted papillomas with dysplasia (C), and inverted papillomas with squamous cell carcinoma (D). (A) Strong and diffuse staining was found in nasal polyps (grade 3). (B) Abundant expression was identified in hyperplastic epithelium of inverted papillomas (grade 2). (C) Slightly staining area was shown in inverted papillomas with dysplasia (grade 1). (D) Scattered staining was shown in malignant transformation areas of inverted papillomas with squamous cell carcinoma (grade 0) (original magnification, x 200).

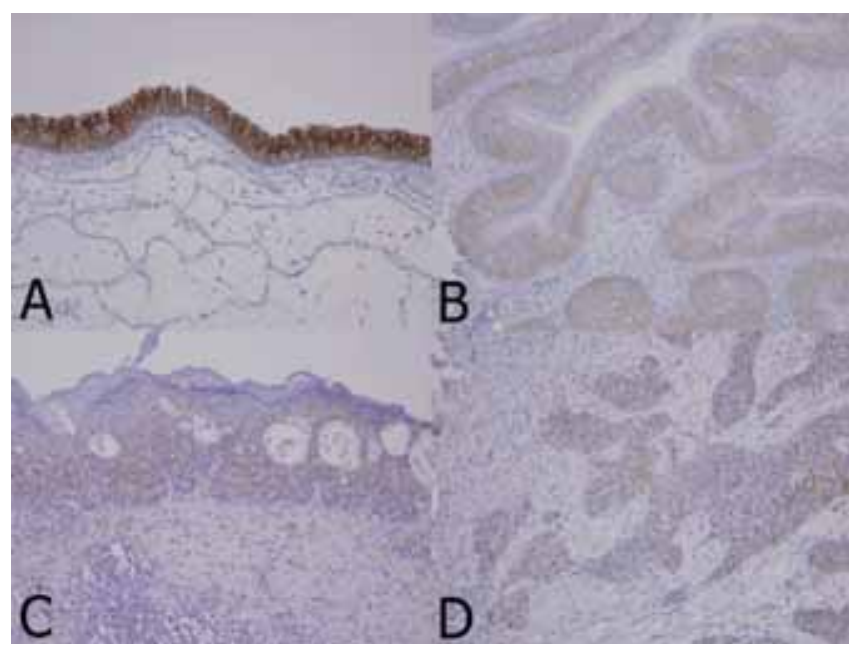

Figure 2. Immunohistochemical stain for $\beta$-catenin. (A) Intensive staining of entire epithelial layer was found in nasal polyps (grade 3). (B) Moderate-to-high stained epithelium was identified in inverted papillomas (grade 2). (C) Slightly staining area was shown in inverted papillomas with dysplasia (grade 1). (D) A little staining area was found in the epithelial region of inverted papillomas with squamous cell carcinoma (grade 0) (original magnification, X 200).

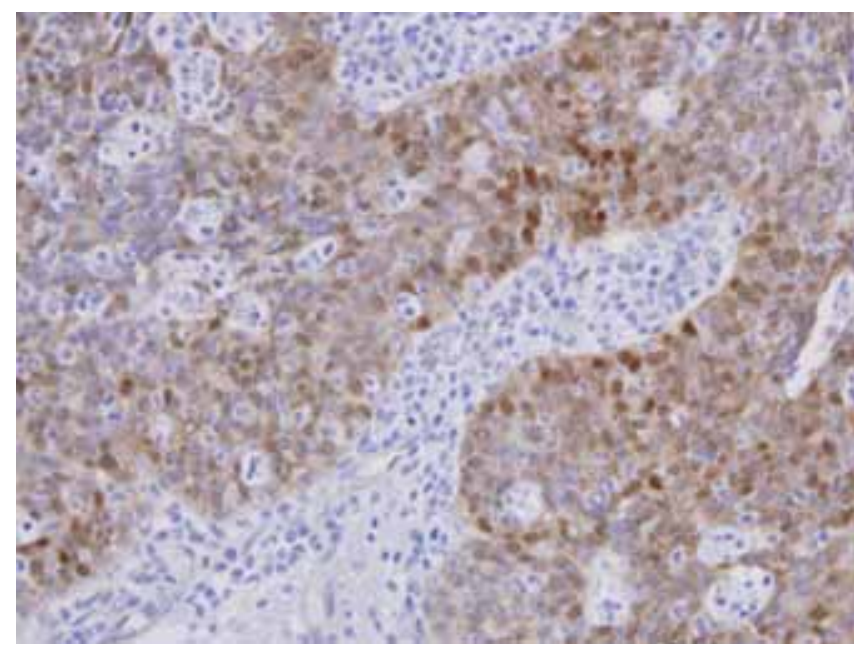

Figure 3. Nuclear $\beta$-catenin staining in inverted papillomas with squamous cell carcinoma (original magnification, X 400).

E-cadherin expression may be an important step in the development and progression of a malignant tumour, such as diffuse gastric cancer, colorectal cancer, squamous cell carcinoma of the skin, and lobular breast cancer ${ }^{(19-22)}$. However, there have been no studies assessing the role of E-cadherin and $\beta$-catenin in malignant transformation of sinonasal IPs. Therefore, we compared the degree of expression of E-cadherin and $\beta$-catenin in NPs, IPs, IPs with dysplasia, and IPs with SCC to identify the role in malignant transformation of IPs.

In the current study, the degree of expression of E-cadherin and $\beta$-catenin in the cell membrane was significantly lower in
IPs with SCC than that in NPs and IPs. In cases of IPs with dysplasia, namely the pre-cancerous lesions of IPs, the degree of expression of E-cadherin and $\beta$-catenin appeared to be lower compared with NPs and IPs. Nuclear $\beta$-catenin expression was positive in $66.7 \%$ of IPs with SCC (12 of 18) in our study. The aberrant localization of $\beta$-catenin in the cell nucleus has been demonstrated in many malignant tumours ${ }^{(23-25)}$. The actions of $\beta$-catenin in signaling pathways, in which the most investigated pathway associated with catenins in humans is the so-called Wnt signaling pathway, have only been partially clarified ${ }^{(1)}$. Without Wnt signaling, $\beta$-catenin is readily phosphorylated and eventually degraded ${ }^{(1,5)}$. The activation of the Wnt signal- 
ing pathway may block the turnover of uncomplexed $\beta$-catenin, resulting in increased cytoplasmic accumulation. Beta-catenin may then associate with translocation to the nucleus, and alter the transcription of target genes ${ }^{(1,5)}$. Mutations of $\beta$-catenin and/or APC have been reported in the colon (26), anaplastic thyroid ${ }^{(23)}$, hepatocellular cancer ${ }^{(24)}$, as well as in malignant melanoma ${ }^{(25)}$. In many reports, these mutations have been shown to be accompanied by nuclear localization of $\beta$-catenin ${ }^{(7,23-25)}$, suggesting that similar mechanisms might be responsible for nuclear $\beta$-catenin expression of IPs with SCC detected in the current study. Thus, the E-cadherin-catenin complex may play an important role in the development of malignant transformation in IPs. Moreover, these biologic markers, reflecting malignant transformation of IPs, may be of great help to the clinician in the management of IPs.

In general, E-cadherin and catenin staining is strong in well differentiated cancers that maintain cell adhesiveness and are less invasive, but is reduced in poorly differentiated tumours, which have lost cell-cell adhesion and show strong invasive behaviour. There are many reports that show E-cadherin is associated with tumour cell differentiation and invasiveness in head and neck and oesophageal cancers ${ }^{(27,28)}$. Therefore, we also determined the correlation of E-cadherin and b-catenin expression with clinicopathological parameters in IPs with SCC. In the current study, the degree of $\beta$-catenin expression was significantly lower in patients with IPs with SCC with a malignant proportion $>50 \%$ compared to patients with a malignant proportion $\leq 50 \%$. However, there was no significant association between the degree of expression of E-cadherin and $\beta$-catenin and clinicopathological variables, such as age, gender, T stage, tumour differentiation, and SCC type (metachronous vs. synchronous). Kutrz et al. ${ }^{(29)}$ reported that decreased E-cadherin, but not $\beta$-catenin expression, was associated with vascular invasion and decreased survival in head and neck SCC. Pukkila et al. ${ }^{(30)}$ showed that nuclear $\beta$-catenin expression independently predicted short overall survival in oropharyngeal and hypopharyngeal SCC. In the current study, there were no statistically significant relationships between recurrence or the survival rate in patients with IPs with SCC and the degree of expression of E-cadherin or $\beta$-catenin in the cell membrane and nuclear $\beta$-catenin. These results may be attributed to the small number of patients with IPs with SCC, which has a better prognosis than invasive SCC of the head and neck.

\section{CONCLUSION}

We demonstrated that the degree of expression of E-cadherin and $\beta$-catenin in IPs with SCC was significantly lower than NPs and IPs. This is the first study to determine the expression of E-cadherin and $\beta$-catenin in NPs, IPs, and IPs with SCC. Decreased expression of E-cadherin and $\beta$-catenin in the cell membrane may be associated with carcinogenesis of IP and help predict malignant transformation in sinonasal IP. Further studies will be necessary to reveal the exact role of E-cadherin and $\beta$-catenin in the malignant transformation of IP.

\section{CONFLICT OF INTEREST}

We don't have any financial disclosure of significant financial interest or affiliations we may have with the manufacturers of products that may be discussed in this article.

\section{REFERENCES}

1. Wijnhoven BP, Dinjens WN, Pignatelli M. E-cadherin-catenin cell-cell adhesion complex and human cancer. Br J Surg. 2000; 87: 992-1005.

2. Wu H, Lotan R, Menter D, Lippman SM, Xu XC. Expression of E-cadherin is associated with squamous differentiation in squamous cell carcinoma. Anticancer Res. 2000; 20: 1385-1390.

3. Farmer I, Freysdottir J, Dalghous AM, Fortune F. Expression of adhesion and activation molecules in human buccal epithelial cell lines and normal human buccal epithelium in situ. J Oral Pathol Med. 2001; 30: 113-120.

4. Takeichi M. Cadherins in cancer: implications for invasion and metastasis. Curr Opin Cell Biol. 1993; 5: 806-811.

5. Hajra KM, Fearon ER. Cadherin and catenin alterations in human cancer. Genes Chromosomes Cancer. 2002; 34: 255-268.

6. Perl AK, Wilgenbus P, Dahl U, Semb H, Christofori G. A casual role for E-cadherin in the transition from adenoma to carcinoma. Nature. 1998; 392: 190-193.

7. Sulzer MA, Leers MP, van Noord JA, Bollen EC, Theunissen PH. Reduced E-cadherin expression is associated with increased lymph node metastasis and unfavorable prognosis in non-small cell lung cancer. Am J Respir Crit Care Med. 1998; 157: 1319-1323.

8. Kuczyk M, Serth J, Machtens S, et al. Expression of E-cadherin in primary prostate cancer: correlation with clinical features. Br J Urol. 1998; 81: 406-412.

9. Tamura S, Shiozaki H, Miyata M, et al. Decreased E-cadherin expression is associated with haematogenous recurrence and poor prognosis in patients with squamous cell carcinoma of the oesophagus. Br J Surg. 1996; 83: 1608-1614.

10. Jian WG, Darnton SJ, Jenner K, Billingham LJ, Matthews HR. Expression of E-cadherin in oesophageal carcinomas from the UK and China: disparities in prognostic significance. J Clin Pathol. 1997; 50: 640-644.

11. Lawson W, Ho BT, Shaari CM, Biller HE. Inverted papilloma: a report of 112 cases. Laryngoscope. 1995; 105: 282-288.

12. Lawson W, Kaufman MR, Biller HF. Treatment outcomes in the management of inverted papilloma: an analysis of 160 cases. Laryngoscope. 2003; 113: 1548-1556.

13. Vrabec DP. The inverted Schneiderian papilloma: a 25-year study. Laryngoscope. 1994; 104: 582-605.

14. Bailey T, Biddlestone L, Shepherd N, et al. Altered cadherin and catenin complexes in the Barrett's esophagus-dysplasia-adenocarcinoma sequence: correlation with disease progression and dedifferentiation. AM J Pathol. 1998; 152: 135-144.

15. Valizadeh A, Karayiannakis AJ, el-Hariry I, Kmiot W, Pignatelli M. Expression of E-cadherin-associated molecules (alpha-, beta-, and gamma-catenin and p120) in colorectal polyps. Am J Pathol. 1997; 150: 1977-1984.

16. De Leeuw WJ, Berx G, Vos CB, et al. Simultaneous loss of E-cadherin and catenins in invasive lobular breast cancer and lobular carcinoma in situ. J Pathol. 1997; 183: 404-411.

17. Batsakis JG. Pathology consultation: nasal (Schneiderian) Papilloma. Ann Otol Laryngol. 1981; 90: 190-191.

18. Hyams VJ. Papillomas of the nasal cavity and paranasal sinuses: a clinicopathological study of 315 cases. Ann Otol Rhinol Laryngol. 1971; 80: 192-206.

19. Koseki S, Aoki T, Ansai S, et al. An immunohistochemical study of E-cadherin expression in human squamous cell carcinoma of the skin: relationship between decreased expression of E-cadherin in the primary lesion and regional lymph node metastasis. J Dermatol. 1999; 26: 416-422.

20. Garcia del Muro X, Torregrosa A, Munoz J, et al. Prognostic value of the expression of E-cadherin and beta-catenin in bladder cancer. Eur J Cancer. 2000; 36: 357-362. 
21. Huiping C, Sigugeirsdottir JR, Jonasson JG, et al. Chromosome alteration and E-cadherin mutations in human lobular breast cancer. Br J Cancer. 1999; 81: 1103-1110.

22. Becker KF, Kremmer E, Eulitz M, et al. Analysis of E-cadherin in diffuse-type gastric cancer using a mutation-specific monoclonal antibody. Am J Pathol. 1999; 155: 1803-1809.

23. Garcia-Rostan G, Gallini G, Herrero A, et al. Frequent mutation and nuclear localization of $\beta$-catenin in anaplastic thyroid carcinoma. Cancer Res. 1999; 59: 1811-1815.

24. Nhieu JT, Renard CA, Wei Y, et al. Nuclear accumulation of mutated $\beta$-catenin in hepatocellular carcinoma is associated with increased cell proliferation. Am J Pathol. 1999; 155: 703-710.

25. Rimm DL, Caca K, Hu G, et al. Frequent nuclear/cytoplasmic localization of $\beta$-catenin without exon 3 mutations in malignant melanoma. Am J Pathol. 1999; 154: 325-329.

26. Morin PJ, Sparks AB, Korinek V, et al. Activation of $\beta$-cateninTcf signaling in colon cancer by mutation in $\beta$-catenin or APC. Science. 1997; 275:1787-1790.

27. Doki Y, Shiozaki H, Tahara H, et al. Correlation between E-cadherin expression and invasiveness in vitro in a human esophageal cancer cell line. Cancer Res. 1993; 53: 3421-3426.

28. Shipper JH, Frixen UH, Behrens J, Unger A, Jahnke K, Birchmeier W. E-cadherin expression in squamous cell carcinomas of head and neck: inverse correlation with tumor dedifferentiation and lymph node metastasis. Cancer Res. 1991; 51: 6328-6337.
29. Kurtz KA, Hoffman HT, Zimmerman MB, Robinson RA. Decreased E-cadherin but not $\beta$-catenin expression is associated with vascular invasion and decreased survival in head and neck squamous carcinomas. Otolaryngol Head Neck Surg. 2006; 134: 142-146.

30. Pukkila MJ, Virtaniemi JA, Kumpulainen EJ, et al. Nuclear $\beta$-catenin expression is related to unfavourable outcome in oropharyngeal and hypopharyngeal squamous cell carcinoma. J Clin Pathol. 2001; 54: 42-47.

Ki-Sang Rha, MD, PhD, Professor

Department of Otolaryngology - Head \& Neck Surgery

Chungnam National University, School of Medicine

640 Daesa-Dong, Chung-Gu

Daejeon, 301-040

South Korea

Tel: $+82-42-280-7698$

Fax: +82-42-253-4059

E-mail: ksrha@cnu.ac.kr

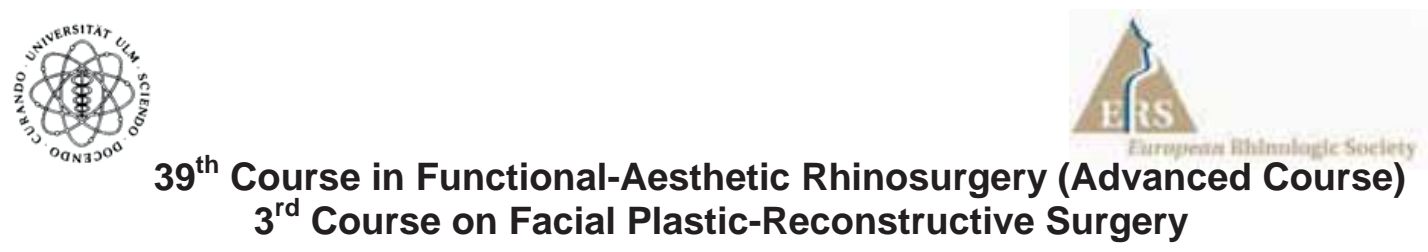

Course Directors: Priv.-Doz. Dr. M. Scheithauer, Prof. Dr. G. Rettinger, Prof. Dr. N. Rotter

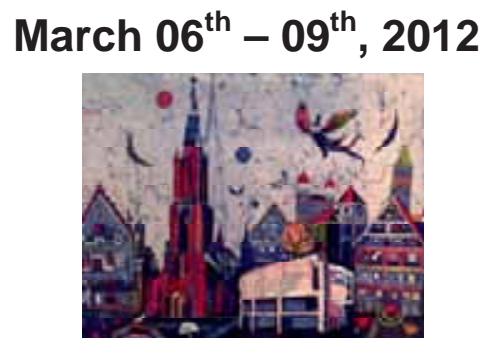

\section{University Hospital of Ulm, Germany}

The international advanced course in functional and aesthetic rhinosurgery is a continuation of the basic course. Knowledge about the dynamic pillar function of the septum and its correction by septoplasty is assumed. The main topics will focus on any kinds of disturbances of form and function like the tension nose, the deviated nose, the saddle nose and cleft lip noses. We will also have lectures on detailed structural problems such as the nasal valve and tip deformities. Most items will be highlighted during live surgery, and again we will offer a "hands-on-dissection" course.

Within the Facial Plastic-Reconstructive Course we focus on typical defects after tumor-resection. We will demonstrate the reconstruction with safe and reliable surgical techniques.

Course fees include food and beverage, course dinner and instruments needed for anatomical dissections.

Information and Registration

Course Secretariat: Mrs. Weis

ENT-Department, University of Ulm

Frauensteige 12, 89075 Ulm, Germany

Online registration: www.uniklinik-ulm.de/hno
Phone: 004973150059710

Fax: 004973150059509

email: course.ent@uniklinik-ulm.de 